\title{
Utilizing Verbally Told Stories for Informal Knowledge Management
}

\author{
Stephan Lukosch • Michael Klebl • Tanja Buttler
}

Published online: 25 February 2011

(C) Springer Science+Business Media B.V. 2011

\begin{abstract}
In knowledge management, the act of telling stories is utilized to capture and convey knowledge. Spoken language is the basis for telling stories. Collaborative audio-based storytelling uses the act of verbally telling stories in groups. In this paper, we explore how to utilize verbally told stories for informal knowledge management. To identify the requirements for an information system that supports collaborative audio-based storytelling, we review the body of knowledge in relation to storytelling and knowledge management. After reviewing the state of the art, we present the CASTing information system. CASTing (Collaborative Audio-based StoryTelling) addresses the identified requirements and supports a process for collaborative audiobased storytelling. CASTing consists of a client application which supports nomadic work as well as a web portal which aims at building a storytelling community. We present CASTing along our process for collecting, structuring, linking and using verbally told stories. We conclude with a report on first experiences as well as an outlook on future directions.
\end{abstract}

Keywords Storytelling · Collaboration · Knowledge management · Verbally told stories

S. Lukosch $(\bowtie) \cdot$ T. Buttler

Faculty of Technology, Policy and Management Systems Engineering Section,

Delft University of Technology, Jaffalaan 5, 2628 BX Delft, The Netherlands

e-mail: s.g.lukosch@tudelft.nl

\section{Klebl}

Department of Vocational Education, WHL Graduate School of Business and Economics, Hohbergweb 15-17, 77933 Lahr, Germany

e-mail: michael.klebl@whl-lahr.de 


\section{Introduction}

Telling stories is not only a given phenomenon of human practice; it is purposefully used as a method or a procedure in different areas of application under the designation storytelling. Collaborative storytelling aims at the development of a common understanding within a group by coordinated narrating activities (when each person contributes his or her own knowledge and his or her own interpretation of a common experience) in order to elicit and disseminate tacit knowledge.

Spoken language is a fundamental and quite natural part of human communication, whereas written communication always requires technical effort. Spoken language is the basis for telling stories, even if in the western industrial societies literary, i.e. written, forms of stories are widely common. However, when considering the increased demand for audio books and the rise of podcasting, a comeback of listening can be determined. By using verbally told stories only, collaborative storytelling can be rooted in everyday's life communication, take advantage of the renaissance of listening and allow stakeholders to capture experiences in-situ.

Consider for example, a company which has the goal to elicit implicit knowledge on project management. Expert project managers like to share their knowledge about best practices for project management as well as lessons learned while dealing with problems in a project. However, often this informal knowledge is not captured and made accessible to novice project managers. The everyday practice in informal knowledge management further shows that this kind of knowledge is usually communicated in form of stories (Brown and Duguid 2000; Stewart 1998). However, these stories are not preserved, accessible and reusable. Furthermore, the stories are not abstracted and the best practices in the stories are not revealed. As a result, the ageing population of expert project managers knowing these stories and capable of telling these stories leads to a gradual loss of implicit knowledge and best practices.

In our opinion, such implicit knowledge can be captured, be preserved and made explicit by means of collaborative audio-based storytelling. In this paper, we investigate how collaborative audio-based storytelling can be supported by an information system and which requirements such an information system has to fulfill.

Following a design science approach (Hevner et al. 2004), we first review the body of knowledge in relation to collaborative storytelling and knowledge management. Based on this body of knowledge, we discuss our research motivation and define our research question. Based on an application scenario, we identify the requirements for an information system that enables audio-based collaborative storytelling. Subsequently, we present a process and the CASTing (Collaborative Audio-based StoryTelling) information system, which are our solution to address the identified requirements. We report on first experiences and discuss how these first experiences will influence future iterations in the development of CASTing. We finally conclude with a view on future developments.

\section{Background}

In this section we will first briefly review the body of knowledge in relation to storytelling in knowledge management and education as well as applications of collaborative 
storytelling and the use of verbally told stories. Based on several insights found in these domains, we then motivate our approach towards a collaborative process based on the verbal telling of stories.

\subsection{Applications of Storytelling in Knowledge Management}

The term knowledge management refers to various approaches for improving how organizations capture, distribute and employ knowledge in order to perform and compete. Amongst these various approaches, storytelling is used as a more or less formalized method. Boyce (1995) offers an account on early research and development in organizational storytelling. Starting from research on stories and myth as a given phenomenon that shape the culture of an organization, e.g. (Clark 1972), storytelling within organization increasingly gained attention as a method to change organizational as well as individual capabilities, e.g. (McWhinney and Batista 1988). More recently, organizational storytelling is connected to the notion of tacit knowledge, i.e. to abilities that are bound to experience, that are neither formalized nor verbalized, and that are initially not reflected.

Storytelling in knowledge management is considered as a suitable method to organize tacit knowledge (Swap et al. 2001). Since this turn from culture to knowledge, a number of authors have devised guidelines or procedures to foster the use of stories and storytelling in organizations. Rules of action have been systematically structured by Ready (2002) or Sole and Wilson (2002). A checklist for efficient stories has been provided by Armstrong (1992), whereas Denning (2000) has described a procedure for the creation of successful stories. Snowden (2000a,b) has offered a comprehensive approach towards the implementation and usage of stories in organizations. However, other authors suggest an approach more focused on the everyday practice of storytelling in organizations, in order to learn from established procedures and thus utilizing embedded and accepted ways of communicating through stories (Brown and Duguid 2000; Stewart 1998).

Considering these various approaches towards storytelling within knowledge management we will focus on key functions of applied storytelling by connecting stories to two main tasks of knowledge management, i.e. the acquisition and the distribution of knowledge:

- With focus on the acquisition of knowledge, storytelling is employed in order to capture knowledge that is possibly neglected by other, rather formal strategies of knowledge management. Storytelling is employed to document experience that otherwise may simply be lost or forgotten. This experience may stem from collaborative work in project groups, from negotiations and transactions with clients or partners, or from individual specialized experts. Best practice, lessons learned, or war stories are common formats for sharing experience through stories. Hence, practitioners like the members of a project team or leaving experts are requested to share their experience by telling stories. These stories may reveal unknown facts, efficient methods, proven solutions and new ideas (Valle et al. 2002), e.g., use storytelling for the recollection of decision in implementation processes, in order to capture insights and best-practices for future decisions. 
- With focus on the distribution of knowledge, storytelling is preferred over other strategies of knowledge management, where personal and social factors of organizational performance are decisive. As opposed to knowledge, stories may focus on attitudes, beliefs, expectations, shared understanding, norms and values. Rich in connotation and context, stories offer a complex, but still coherent and convincing explanation of behavior, events, rules and routines. Therefore, stories are told within an organization or within a group in order to align individual behavior with shared aims and objectives. These stories concerning personal or social issues emerge informally as a part of everyday practice in organizations or are purposefully designed and managed. Schäfer et al. (2004a,b) report the application of storytelling for team-building, raising team awareness through collectively remembered episodes

- With regard to both the acquisition and the distribution of knowledge storytelling is applied in order to capture and communicate knowledge that is hard to document, i.e. tacit knowledge. Here, stories that are told within a community of practice convey capabilities amongst the members of a group that share a common interest, even if facts, methods, solutions and ideas are not made explicit beside the story itself. Listening to stories told by experienced members in a community of practice may foster knowledge, skills, attitudes and competencies, even if facts, methods, solutions and reasons are not made explicit (Acosta et al. 2004), e.g., present a multimedia tool which addresses the externalization of implicit knowledge while (Linde 2001) puts an emphasis on social knowledge that is communicated through stories, i.e. on knowledge concerning group identity and performance.

Concluding from the above, insights that are captured, distributed and employed by the use of stories are considered specific. Either they are hard to discover and collect, or they address rather emotion and motivation than reason, or they are implicit, complex and bound to context. Consequently, also other disciplines related to knowledge management give attention to storytelling as a method. For example, in requirements engineering for information systems stories told by users relate to the notion of scenarios and use cases, both utilized as models for the elicitation as well as the negotiation of the stakeholders' needs (Cheng and Atlee 2007; Lukosch et al. 2009). Storytelling was early introduced into requirements engineering and made applicable through methods for extracting, for structuring, and for systematically modeling requirements and specifications (Faro and Giordano 1998). In addition to this unidirectional approach, the use of Storycards in eXtreme Programming (XP) (Beck 1999) aims at facilitating communication between software engineers and users, as suggested by Keil and Carmel (1995). The narrations of the latter as ordinary people contribute to a deepened understanding of knowledge in the targeted domain. This way, the social roles of layman and experts exchange mutually, since users are considered as experts in their field of practice. Laporti et al. (2009) present an integrated approach for the elicitation and the negotiation of system requirements that employs group storytelling.

Similar to requirements engineering, business process engineering has become a further domain for the application of storytelling. Santoro et al. (2009) employ the group storytelling approach for the acquisition of knowledge on past, present and future business processes negotiating between the concerns of different stakeholders. 


\subsection{Applications of Storytelling in Education}

Stories told to learners provide a complex situated context and thus foster meaning, transfer, and motivation. Learners are asked to solve problems that derive from a story by the use of new knowledge and skills. Here, storytelling is an essential part of situated learning within the approach of anchored instruction, as exemplified in the Jasper Series (Cognition and Technology Group, Vanderbilt University 1992).

In learning environments where learners are asked to perform authentic tasks that are often framed by a story, case studies offer knowledge and skills that are required for proficient practice. This use of storytelling, here, connects to the notion that the knowledge of experts is captured in stories. This approach is referred to as learning in a goal based scenario (Schank et al. 1994). Furthermore, the use of storytelling in education prompts learners to tell stories themselves. By telling stories students make sense of experience, develop understanding of complex issues, and share insights and meaning (Nyrgen and Blom 2001). Thus, storytelling is connected to cognitive activities of learning, such as recalling, comparing, generalizing, deducing, or classifying. In addition, storytelling tools like KidStory (Bayon et al. 2003) with a tangible user interface allow for group interaction, taking storytelling activities from the desktop computer to physical activities in mixed reality environments. With regard to multiplayer games in virtual worlds, storytelling allows for the training of social skills (Schäfer et al. 2004a,b).

In verbal or written stories, cognitive, behavioral or social activities of learners become visible. Hence, they become available for reflection and discussion. Viégas et al. (2004) revealed the impact of visualizing the e-mail history for the participants in e-mail conversations: Their visualization of evolving social relationships, made visible from email exchanges, prompted users to tell stories about their social network. At present, there are first attempts to foster self-reflective storytelling by visualizing artifacts based upon activities in social networks (Zalinger 2009).

The need for reflection on what is being learned is essential for the model of reflective learning through storytelling proposed by (McDrury and Alterio 2003). This approach offers a classification for the stages in a storytelling process and describes a storytelling procedure using five phases: story finding, story telling, story expanding, story processing, and story reconstructing. For this approach, two points deserve a specific mention:

1. The storytelling process proposed by (McDrury and Alterio 2003) is conducted in a collaborative setting. Thus, learners tell and listen to stories told by other learners, as well as groups of learners are asked to develop one shared story.

2. Prompting learners to tell stories indicates a shift towards a learner centered approach. Once more, the social roles of layman (learners) and experts (teachers) mutually exchange. Learners are encouraged to take responsibility for their learning.

The latter point leads to approaches to storytelling targeting participation or qualification for participation. These approaches are primarily employed in media, politics, education and social networks. A sample approach for the use of storytelling to empower people can be found at the Center for Digital Storytelling, a non-profit 
organization offering training, project development, and research. Here, stories told by ordinary people are made public through the use of multimedia and hypermedia technology. For this digital storytelling approach, advocated by Lambert (2008), a procedure for a several day event has been devised where people can script and create their stories using multimedia software applications. This process comprises seven key components for constructing a story: point of view, dramatic question, emotional content, voiceover, soundtrack, economy and pacing. It has two major objectives: First, individuals are encouraged to develop a personal point of view by remembering and narrating their biography. Second, starting from individual stances, groups are prompted to come to collective stories in order to foster community building and participatory problem-solving. Digital storytelling follows the approach of advocacy oral history, which takes a critical stance towards traditional historiography. Advocacy oral history aims to reveal the lives and the needs of ordinary people as opposed to the view of the power wielders (Hampton and Fayer 1990). At present, participatory approaches to storytelling are promoted by grassroots media (Gillmor 2006), e.g. by the concept of community radio, i.e. open channels on local radio (Howley 2000).

\subsection{Collaborative Storytelling}

Drawing on the above approaches towards the use of storytelling in different fields, the relation of stories and knowledge has to be analyzed under two aspects. First: Obviously, there is knowledge that can be captured by storytelling in order to explain it in the form of facts, methods, thoughts and reasons. Furthermore, there are insights that can be conveyed by the use of stories, employing distinctive mechanisms unique to the narrative. However, there is a possibility that knowledge embodied in stories is of a different kind. Narrative knowledge is of a specific rationality, since it is bound to context, historicity, culture and the social relation between the teller of a story and his or her listeners. As a result, a conversion of narrative knowledge into rather formal forms of knowledge may be difficult, if not impossible (Schreyögg and Geiger 2005).

Secondly, the telling of stories is a social activity. As such, storytelling can be understood as well as employed only by consideration of the context in which stories are being told. Focusing either on the teller (and the telling), or the story itself, or the listener (and the listening), appears to be insufficient. Narration is a specific form of discourse. Here, the social relations between the persons taking part in the process are specified, e.g. in the form of roles as initiators, producers, co-producers and recipients. This results in symmetrical or asymmetrical relations. While in symmetrical relations all participants have equal means to influence the course of communication, abilities are unequally distributed in asymmetrical settings. In the field of application for storytelling three perspectives on these relations can be differentiated:

- top-down: Approaches to storytelling in knowledge management which are related to corporate goals often presuppose an asymmetrical relationship between the tellers of stories and their recipients, where the storytellers have means to define knowledge. In addition, the utilization of the storytelling addressing knowledge transfer or dissemination of information in educational contexts equally differentiates social roles when stories are told for learners, not by learners. 
- peer-to-peer: Approaches focusing on discourse are predominantly found where collaborative storytelling is used pedagogically. This is where students invent and tell stories in order to acquire or construct knowledge in the learning process. However, these forms of discourse are also shown in areas of knowledge management.

- bottom-up: It seems that forms of storytelling addressing facilitation, advocacy, and empowerment are suited for pedagogical, public or political issues. However, even in knowledge management the need to encourage those who have fewer possibilities for communication to express their knowledge and their requirements can be stated. Alleged laymen, e.g. customers of products and services, users of a technical product, and even employees in a company, should be considered to be experts for the area of application. As such their narrations contribute to a deepened understanding of knowledge in the target domain.

Apparently, the application of group storytelling in areas of knowledge management favors peer-to-peer and bottom-up approaches: Perret et al. (2004) report on narrative reflection on completed projects. As already mentioned, (Valle et al. 2002) use storytelling to recall decision processes in work groups, while (Schäfer et al. 2004a,b) apply storytelling to team building. All these approaches rely on the collective remembering of episodes and configurations of the group's history. Shen et al. (2002) examined the role of artifacts in this process of collective recall, allowing for the structuring of pictures and photographs on a tabletop display. Carminatti et al. (2006) present an experimental comparison of knowledge recall, contrasting interviews with group storytelling and investigating the impact of tool support in group storytelling. As a result of this study, better result for group storytelling compared to interviews can be stated. However, the tool support (employing TellStory) achieved some of the expected advantages, while other problems appeared.

The thoughtful consideration of the social context in which stories are being told adopts an approach that is focused on the everyday practice of storytelling. As already stated, adequate approaches connect to accepted procedures of communicating through stories, in order to utilize and enhance them rather than imposing restrictive regulations (Brown and Duguid 2000; Stewart 1998).

Narration as a specific form of discourse can be observed in closely connected groups, e.g. in work teams, communities, or families. There, the act of telling stories in groups ties to the everyday experience of discussing collectively remembered episodes. Here, narrative structures do not develop exclusively as a creation of an autonomously narrating person, but are formed by demands, additions, references, interpretation offers and much more from listening persons, who become co-tellers.

In their studies of family communication, (Ochs and Capps 2001) draw attention to narrative activities, where stories are formed collaboratively. In the common situation of talking about an event in a group, often neither teller nor listener are clearly defined roles. On the contrary, all participants contribute to the story that is being told in the roles of initiators, co-tellers, commentators, producers. Ochs and Capps develop a dimensional model that describes this form of stories. Their model posits five distinctive features, i.e. tellership, ellability, embeddedness, linearity/temporality, moral 
stance, in order to distinguish collaboratively told stories from stories told by a single teller:

- Tellership refers to the degree of involvement of multiple people in the re-counting of a story. While a traditional view of storytelling is focused on a single teller, a shared tellership characterizes collaborative storytelling.

- Tellability indicates the level in structure that a story integrates. Stories high in tellability are well-structured, using plot devices like exposition, characters, sequences, or turning points. Low tellability characterizes stories that are hard to convey in common narrative structures.

- Embeddedness specifies the relation between the story itself and the situation of narrating the story. Usually stories are told detached from the social setting of storytelling, and hence can be easily told in other situations. However, stories told in groups refer to the immediate setting, including present social interaction within the story.

- Linearity and temporality expose the sequential, logical and temporal order of events in a story. Traditionally, a story is expected to follow a single, closed and causal path in order to make sense. On the contrary, stories told in groups often comprise unordered events, alternative directions, and even contradictions.

- Moral stance suggests a valuation that is included in a story and refers to the degree of decidedness for values being conveyed through the story. Stories told in groups discuss a moral stance rather than denoting it.

Ochs and Capps (2001) work from the premise that stories are shaped within the range of possibilities described by these five dimensions. On the one extreme, there are stories told by one active teller. These stories are well structured, detached from the situation of storytelling, linear and temporal organized and have a certain and constant moral stance. On the other end of these continua, there are stories in the making, with multiple tellers, low in structure, embedded in surrounding discourse and activity, open in temporal and causal order, and with a fluid moral stance.

According to Ochs and Capps (2001), research in linguistics as well as in social science is predominantly focused on the first type of stories. An obvious reason for that is that stories in the making are hard to capture for investigation, whereas complete stories are well suited for both research and utilization. Furthermore, the dimensional model of Ochs and Capps offers an approach towards the narrative process in collaborative storytelling. Starting from considering the narrative process as a dialogue, storytelling in groups allows for the iterative formation of a story, influenced by those participating in the process of storytelling. This applies to the single situation of storytelling, where questions, comments, demands, and additions form the story. In addition, groups like families or work teams tend to retell important stories time after time. By retelling stories over and over, the stories gradually move from the one end of the proposed dimensions to the other. Hence, a first report on a shared experience is low in structure, organization, and moral stance, and will become well tellable, detached, and determined in valuation after a number of iterations in collaborative retelling. 


\subsection{Comeback of Listening}

Recent developments in everyday media consumption, e.g. the risen demand for audio books (Philips 2007) and the rise of podcasting (Hein and Jakuska 2007), show a comeback of listening. Audio books for both fiction and non-fiction became commonplace at book resellers as recently as the end of the twentieth century. Before that, they were rather positioned in a niche market. In 2004, the term Podcast was introduced for a series of audio files that are available through web syndication. Most commonly, users listen to podcast episodes using digital audio players like the iPod. Podcasts are a serial form of audio on demand. Similar to episodes of an ongoing radio programs, they focus on specific issues or areas of interest.

Considering this comeback of listening, we now draw attention to another essential factor for collaborative storytelling: In everyday life, people verbally tell and listen to stories in groups.

Melnik and Maurer (2004) have drawn the attention to the importance of verbal communication in everyday's work processes when considering knowledge sharing in agile software engineering. Here, verbal communication is usually not captured during the work processes. The knowledge in the verbal communication tends to be informal and thus is part of background communication (Randall et al. 1995). Such background communication results in local knowledge, which is not shared, nor structured or systematically utilized.

Spoken language is a fundamental and quite natural part of human communication, whereas written communication always requires technical effort. Similarly, iconic communication, using graphics, pictures or moving images is based on specific media, even if these are paper and pencil.

Spoken language is the basis for telling stories, even if in the western industrial societies literary, i.e. written, forms of stories are widely common. Regarding verbal versus written communication, frequently a differentiation between oral and literal cultures is made. This differentiation regularly refers to (Ong 1982), who identified unique features of thought, reason, knowledge, rhetoric, and tradition in societies that are predominantly based on oral communication.

In oral cultures, the majority of the population has no access to technologies of literacy. Writing and print is not available in everyday life. And even if people have basic writing and reading skills, most parts of public and private life, like trade, politics or education, do not rely on literacy. However, there are several fallacies based on this differentiation:

1. The definition of oral cultures through the absence of literacy is misleading. The oral tradition of thought and knowledge is not less developed compared to written communication; it is just different. Oral societies have their own means to extend human abilities in thought, communication and interaction. To convey knowledge in these ways can be more appropriate to a given context than literal means. In spite of that, literal societies tend to judge oral societies as subordinate, not reflecting an implicit ethnocentric bias.

2. Orality and literacy are modes of communication which are predominant in a specific society at a certain time. Nevertheless, verbal communication is still vital 
and essential in literal societies. In addition, contemporary oral societies use written communication for specific purposes. For example, Ong (1982) examined the interaction of orality and literacy in communication that is modified by the use of electronic media in his study on secondary orality. Since western industrial societies concentrate on literacy in all areas of public life, like economy, politics, education, including science, verbal communication is given relatively little attention. Consequently, only few approaches in applied social science and economics focus on oral communication. This also applies to knowledge management and storytelling.

Hence, we focus on verbally told stories, in order to connect to the very root of storytelling and in order to utilize this commonly shared practice for informal knowledge management.

\section{Research Motivation and Question}

Considering the body of knowledge as discussed in the previous section, we can now connect four distinctive features of knowledge management, storytelling and verbal communication:

1. Considering narrative knowledge to be of a specific rationality, i.e. bound to context, historicity, culture and the social relation between the teller and the listeners, relates to the oral tradition of thought, reason, knowledge, methods, and ideas. In order to connect both the acquisition and the distribution of knowledge in a community of practice, a transformation of concerns issued in verbal communication to formal statements by means of written communication would break this specific way to convey knowledge through stories.

2. As a fundamental and quite natural part of human communication, spoken language offers potential for non-directive communication. Since verbally narrating, commenting, questioning, or contradicting require less effort than devising a written statement, audio-based storytelling addresses requirements arising from approaches focusing on discourse (peer-to-peer) or aiming at facilitation, advocacy, and empowerment (bottom-up).

3. Narration as a specific form of discourse can be observed in closely connected groups, e.g. in work teams, communities, or families. Especially, in everyday's work practices verbal communication plays an important role for informal knowledge exchange. However, such communication is not shared, nor structured or systematically utilized. In order to utilize this form of discourse systematically, we address collecting, structuring and connecting of verbal statements.

4. Storytelling is connected to cognitive activities of learning, such as recalling, comparing, generalizing, deducing, or classifying. Often storytelling is used to foster reflection in educational scenarios. When considering scenarios with multiple tellers, stories are first low in structure, embedded in surrounding discourse and activity, open in temporal and causal order, and with a fluid moral stance. After a number of iterations of reflecting and retelling, stories become well tellable, detached, and determined in valuation after a number of iterations in collabora- 
tive retelling. Thus, collaborative storytelling promises to support the elicitation of best practices in shared stories.

Considering our introductory example of a company which has the goal to elicit implicit knowledge on project management, we are of the opinion that a collaborative audio-based storytelling approach helps to stop the gradual loss of implicit knowledge and best practices which is due to the ageing population of expert project managers:

- By using verbally told stories the storytelling can be embedded in everyday's work practices of verbal communication and knowledge exchange.

- By using a collaborative approach, the best practices captured in the stories can be revealed.

Such an approach of collaboratively retelling important stories time after time in a group needs to be supported by a tool for collaborative editing. However, while tools for the collaborative editing of textual information are well-known and widely available, devices for recording and collaboratively editing audio are not established. Here, we identify the need for a procedure and supporting information systems that are related to the accepted practice of communicating through stories in groups in order to enhance and utilize the process of shaping shared stories as described above. In the following, we thus address the research question which requirements such an information system has to fulfill and how such an information system has to be designed in order to support a collaborative audio-based storytelling approach.

\section{Requirements Analysis}

In the following, we determine the requirements for supporting collaborative audiobased storytelling with a scenario on eliciting implicit knowledge on project management. Expert project managers often like to share their knowledge about best practices for project management as well as lessons learned while dealing with problems in a project. The everyday practice in informal knowledge management shows that this kind of implicit knowledge is often told in form of stories (Brown and Duguid 2000; Stewart 1998) and thereby communicated from experts to laymen.

The knowledge in these stories is not preserved, accessible and reusable. The ageing population of expert project managers knowing these stories and capable of telling these stories lead to a gradual loss of this implicit knowledge. Although expert project managers like to share their knowledge, they do not have time to constantly tell their stories to young and future project managers. Storytelling will allow project managers to preserve and reveal their tacit knowledge in form of stories. By capturing the knowledge in form of audio stories, these stories can be tagged, searched, and reused.

Collaborative storytelling will allow project managers to contribute to and amend the stories of other project managers. According to Ochs and Capps (2001), collaborative storytelling follows a specific form of discourse in which stories converge towards a constant moral stance. As the captured knowledge within such stories is easily accessible and reusable within other stories, collaborative storytelling will enable the cross-project fertilization in the form of shared implicit knowledge. 
This scenario serves perfectly for informal knowledge management processes. To enable collaborative storytelling and thus cross-project fertilization the following initial requirement has to be fulfilled:

R1: Provide user and project management functionality.

To collaboratively create audio-based stories, project managers first have to record their stories. Still, these audio recordings are then only available to them personally. Still, project managers need support for sharing their audio recordings and creating a shared audio database from which stories can be created collaboratively:

R2: Offer a shared workspace for each story project, in which users can share and manage audio recordings.

In order to facilitate discourse and thus move from a fluid moral stance to a constant one, project managers must be able to contribute to, amend and comment the stories of other project managers. Such contributions have to be placed directly at the point of interest and not at the end of an audio recording. One approach could be to cut the audio recordings into small parts and then mix these audio recordings to capture the new story. This approach leads to a huge amount of audio recordings. The content of such a growing audio repository is difficult to assess and keep in mind when collaboratively telling a story. Thus, to avoid cutting the audio recordings and thereby creating a huge amount of smaller audio recordings, the following requirement has to be met:

R3: Enable users to set marks in the audio recordings which can be used to link other audio recordings.

Such marks allow project managers to specify the exact point of time from where other audio recordings can be referenced. The marks thus allow project managers to link any contribution to the specific points of interest in the audio recording. Thereby, project managers can collaboratively specify stories which consist of audio recordings, marks, and references between marks and audio recordings.

The links between the different audio recordings allow the project managers to construct parallel threads in one story. These parallel threads can be seen as different alternatives of one story. Principally, it is possible to distinguish between linear and non-linear stories (Spaniol et al. 2006). In a linear story there is exactly one thread. A non-linear story can have several parallel threads. Such non-linear stories can be compared with the approach of retelling a story over and over again. But compared to the traditional approach of retelling a story an information system is capable of capturing the different alternatives of a story which is told over and over and again. Thus, such non-linear stories will also gradually move from a simple structure and a fluid moral stance towards a well structured story and a constant moral stance. In relation with our scenario, project managers will first reveal stories that are embedded in their surrounding and activities. By listening to and reflecting on the contributions of other project managers, the stories will evolve and become mature. To support project managers in creating such linked stories, the following requirement has to be met:

R4: Facilitate the creation of links between marks and audio recordings for specifying non-linear stories. 
These stories should not only be available to the group who has created it. Instead there should be a possibility to share the selected threads to a wider community. Podcasts are the current de-facto standard to share audio recordings and stories. For that purpose the following requirement has to be met:

R5: Support the selection of single threads in a non-linear story and its export as podcast.

The concept of non-linear stories allows project managers to create alternative stories and to add comments to existing stories. However, performing complex modifications on a shared story usually takes time and requires cognitive effort on the part of the storyteller. In some cases, e.g. when recording comments or amendments or making experiences relevant for the current story, project managers might have no access to the Internet. Still, these project managers might want to capture the experience insitu and record it. To use the recorded audio clips in the shared story, the following requirement must be met:

R6: Support nomadic work on the shared story.

This kind of nomadic work increases the probability of conflicting changes. To discard conflicting changes is inappropriate, since its originator has already spent much effort in performing the change. Hence, the following requirement must be met:

R7: Support users in synchronizing their results.

To support the convergence of non-linear stories from a simple structure and a fluid moral stance towards a well structure and a constant moral stance, the project managers in our scenario need to be able to discuss the different story threads and create a shared understanding of the story which is being told. To select the final version of a story, i.e. one thread of the non-linear story, the project managers need to be able to vote on different threads and finally publish and share such a thread with other project managers. The latter will be a prerequisite for fostering cross-project fertilization. As audio as a medium is not easily accessible, the cross-project fertilization can even be improved by offering additional functionality to assess the content of a podcast or audio clip. This can be achieved by supporting tagging and search functionality. Considering the above affordances the following requirement emerges:

R8: The audio-based collaborative storytelling support has to offer a community platform which allows users to share, publish, discuss, comment, vote, tag, search and reuse audio stories.

The following Table 1 summarizes our requirements for an information system that enables collaborative storytelling by utilizing verbally told stories.

\section{Related Work}

The previous section identified the requirements for an information system which supports our research motivation and question. In this section, we consider existing storytelling tools and discuss whether these tools are suitable to answer our research question. 
Table 1 Summary of requirements

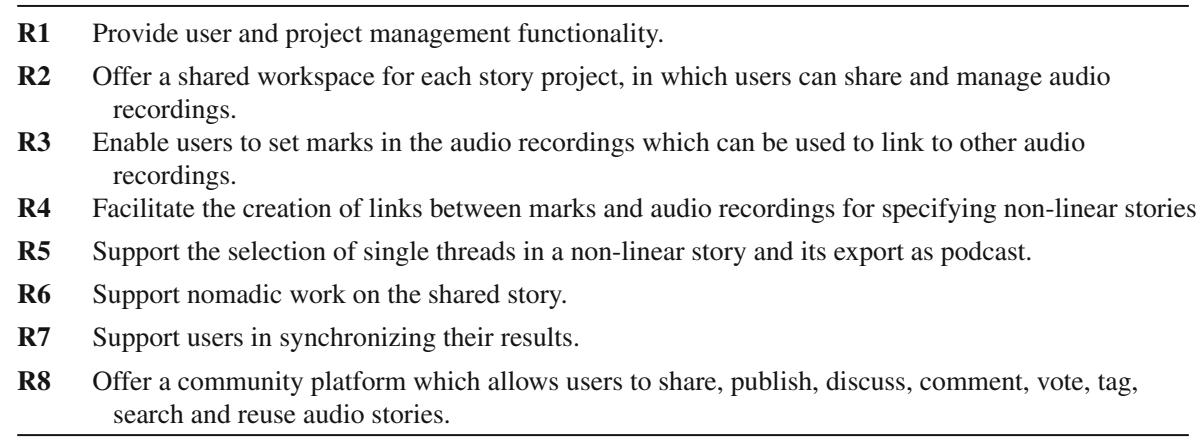

There exist quite a few tools that support users in collaboratively creating stories. StoryMapper (Acosta et al. 2004) supports groups in telling a story and structures the collaboration process by assigning different roles to the members of the group. A story is modeled as conceptual map. Each node in such a conceptual map can be linked to different media artefacts. TellStory (Perret et al. 2004) is a collaborative application that supports groups in creating text-based stories. PhotoStory (Schäfer et al. 2004a,b) uses storytelling to increase the awareness in the group about its external presentation but also its social activities. For that purpose, the group can create stories that consist of a series pictures with corresponding subtitles. VoiceThread (2009) supports groups in creating sequences of images or video clips. Users can add textual or audio based comments to these multimedia artefacts and hence create a digital story. Compared to our requirements, none of these tools focusses on audio-based stories for informal knowledge management and supports a gradual refinement by commenting and amending the existing story. Summing up, the above tools violate R2, R3, and R4.

Apart from the above-mentioned tools that explicitly focus on collaboratively creating a story, the following tools focus on the creation of stories in general. MIST (Spaniol et al. 2006) supports the creation of non-linear multimedia stories. Users can integrate text, images, audio and video recordings in their story. Hence, it is also possible to create stories that solely consist of audio recordings. However, MIST does not focus on collaboration. Although different users can edit the same story, MIST does not support the users when synchronizing parallel changes or working nomadically. Hence, MIST violates R6 and R7.

iTell (Landry and Guzdial 2006b) makes use of a process that consists of four steps. This process allows users to create a text-based story which can be enriched with additional digital media items. Although Landry and Guzdial (Landry and Guzdial 2006a) consider collaboration as one of the fundamental activities when creating a story, iTell does not support it and thereby violates our requirements R2, R3, and specifically R6 and R7.

Röber et al. (2006) introduce the concept of Interactive Audiobooks. Interactive audiobooks combine non-linear audio-based stories with interactive elements as known from computer games. However, such audiobooks are not created collaboratively and again $\mathrm{R} 6$ and $\mathrm{R} 7$ are violated. 
StoryWriter (Steiner and Moher 1992) supports the creation of text-based stories that can be illustrated with images. For creating such a story, StoryWriter supports authors with a rule-based system, which, e.g., keeps track of the interaction among the different characters in the story or even generates text. But again, StoryWriter is not a collaborative application and does not support audio-based stories. Hence, R2, R3, and specifically R6 and R7 are violated.

The above discussion shows that there is currently no sufficient support for collaboratively creating an audio-based story. In addition, none of the discussed tools focuses on establishing an online community as a place for ongoing story exchange and evolution. Thus, R8 is not met as well. In the following section, we show how we address the requirements which we have identified for a tool and a process for collaborative audio-based storytelling.

\section{Approach}

Our approach consists of a storytelling client application which supports the collaborative creation of audio-based stories, a web portal which offers the functionality to publish podcasts and discuss, comment, vote, tag, search and reuse audio stories, and a server which offers the functionality to manage and maintain the shared data. Together these three major components form the CASTing information system.

Figure 1 highlights the main components of the resulting CASTing system architecture. The CASTing web portal and the server are based on Liferay-Enterprise Open Source Portal (2009). The CASTing client application accesses the shared resources on the server via the Tunnel Servlet, whereas the web portal accesses these via a Struts Servlet (Struts Framework 2009). At the server, both servlets can access the Liferay portal logic as well as the Storytelling Kernel which encapsulates the process functionality via an embedded Spring (Spring Application Framework 2009) layer. Finally, the Liferay portal logic and the storytelling kernel provide access to the shared resources which are stored in a Java content repository and a MySQL database. The Java Content Repository is used to store and manage the shared files, i.e. audio clips and documents, whereas the MySQL database is used to manage the application-specific shared data.

Table 2 shows the collaboration process which is supported by the CASTing client application. The collaboration process can be divided into two stages: Setup and Storytelling. The collaboration process is a direct result of the requirements R1-R5. The four steps of the storytelling process stage may be executed in any non-sequential order. It is possible to skip steps or to return to steps in the process. Thereby, we support a free development of the story. When, e.g., linking audio recordings the need for an additional audio clip can become obvious. This clip can be added to the shared audio database, segmented, and then directly be linked.

In the following, we first describe the different process steps as supported by the CASTing client application, before we introduce our web portal in more detail.

\subsection{Creating a Project Team}

To collaboratively create a story, users must be able to build groups and define a common project (R1). As our web portal is based on Liferay-Enterprise Open Source 


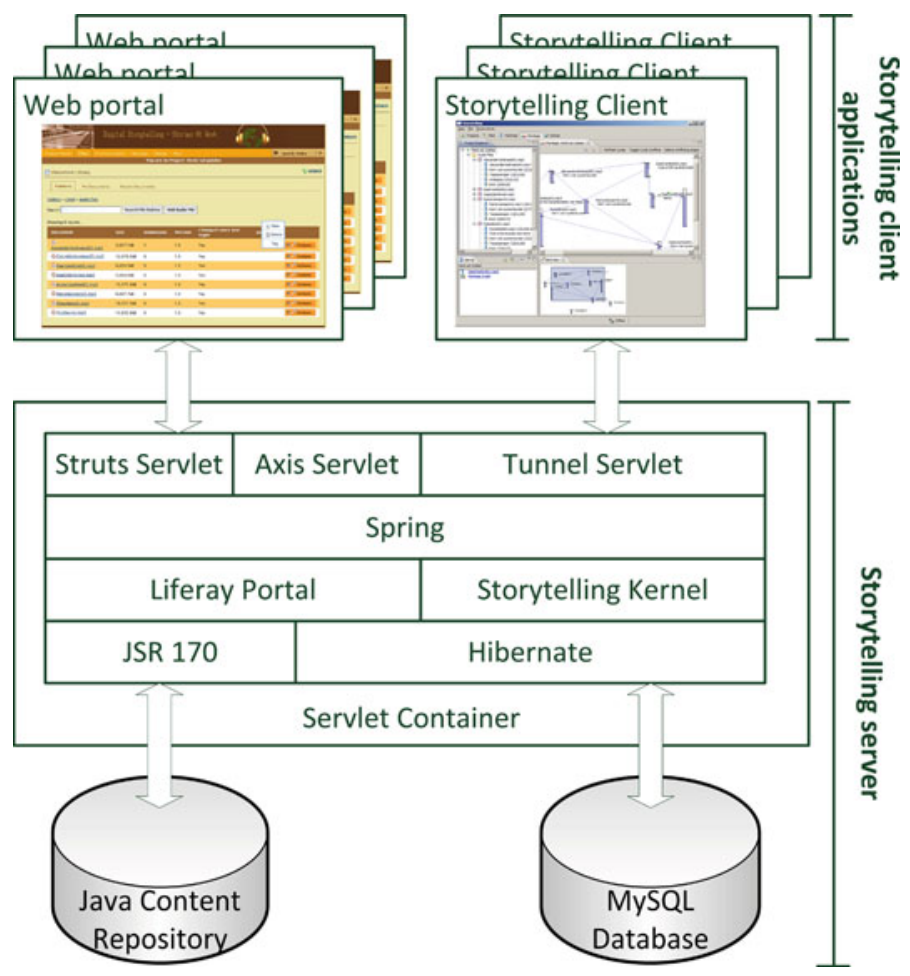

Fig. 1 Overall system architecture

Table 2 Collaboration process supported by the CASTing client application

\begin{tabular}{|c|c|c|c|c|c|}
\hline \multirow{2}{*}{$\begin{array}{l}\text { Phases } \\
\text { Steps }\end{array}$} & \multirow{2}{*}{$\begin{array}{l}\text { Setup } \\
\text { Creating a } \\
\text { project team }\end{array}$} & \multicolumn{4}{|l|}{ Storytelling } \\
\hline & & $\begin{array}{l}\text { Adding audio } \\
\text { recordings }\end{array}$ & $\begin{array}{l}\text { Segmenting } \\
\text { audio } \\
\text { recordings }\end{array}$ & $\begin{array}{l}\text { Linking audio } \\
\text { recordings }\end{array}$ & $\begin{array}{l}\text { Publishing a } \\
\text { story }\end{array}$ \\
\hline Goals & $\begin{array}{l}\text { Building a } \\
\text { group; } \\
\text { creating a } \\
\text { vision of a } \\
\text { story }\end{array}$ & $\begin{array}{l}\text { Sharing } \\
\text { background } \\
\text { information, } \\
\text { audio clips, } \\
\text { comments } \\
\text { and } \\
\text { reflections } \\
\text { with the } \\
\text { group }\end{array}$ & $\begin{array}{l}\text { Finding } \\
\text { interesting } \\
\text { parts of an } \\
\text { audio clip }\end{array}$ & $\begin{array}{l}\text { Connecting } \\
\text { the parts; } \\
\text { creating a } \\
\text { non-linear } \\
\text { story }\end{array}$ & $\begin{array}{l}\text { Selecting a } \\
\text { story out of } \\
\text { personal and } \\
\text { general } \\
\text { interests }\end{array}$ \\
\hline Results & $\begin{array}{l}\text { Shared } \\
\text { workspace, } \\
\text { project }\end{array}$ & Shared resources & Shared markings & Montage & Story \\
\hline
\end{tabular}

Portal (2009), it reuses the provided functionality to request a login. Within the web portal users can create new projects, invite other users to join projects, or access the shared data within such a project. With the same login, users can connect from within 
the CASTing client application with the storytelling server to perform the same actions as well as to synchronize their local projects with the most recent versions at the server.

\subsection{Adding Audio Recordings}

In our second process step, users share their audio recordings for the story in the project workspace (R2). This can be done via the web portal as well as via the client application. The client application has the advantage that it supports all process steps as well as the possibility to work nomadically and share the results when connected again.

For each audio recording, users can enter a textual description or even a transcription. This information can be used to search for audio recordings in the shared audio database. All audio recordings and its versions are managed by the server and stored within the JCR (cf. Fig. 1). Thereby, the server supports the creation of a shared audio repository which can be viewed via the web portal as well as the client application.

\subsection{Segmenting Audio Recordings}

In this process step, users can segment the audio recordings by setting marks (R3). Each mark is defined by a name and a exact time position in the audio recording. Apart from this information the client application also stores who created the mark, when the mark was created, and when and by whom it was last modified. To set a mark, users can listen to the audio recording while its wave form is visualized. This allows users to exactly place a mark in the audio recording.

The marks in an audio recording can later on be used to define links between audio recordings as they can serve as starting or end point for a link. By including marks in the audio recordings these have not to be split into separate parts when only a part is necessary for a story. Additionally, the marks are shared by all users that have access to the shared repository which supports a collaborative segmentation and adds further value to the shared audio database.

Apart from the explicit marks which can be set by the users, each recording includes two implicit marks: the start and the end of the audio recording. These marks are set when the audio recording is imported into the shared workspace.

Figure 2 shows the marking perspective, i.e. the user interface layout, of the CASTing client application and illustrates this process step. The left part of the screenshot shows the project explorer which shows the different projects in which the user participates and the project content, i.e. the corresponding audio recordings, documents, as well as the already proposed stories. Currently, the project explorer shows audio recordings from the 'Henk Sol Jubilee' project (cf. Fig. 2). Within this project, faculty members provided stories on their personal experiences with Henk Sol. The upper right part of the screenshot shows the list of marks for the selected audio recording. The lower right part shows the wave form of the selected audio recording. It allows users to listen to the recording and to define new marks by double-clicking on the point of time where they want to include a new mark. Existing marks can be dragged and dropped to a new position. 


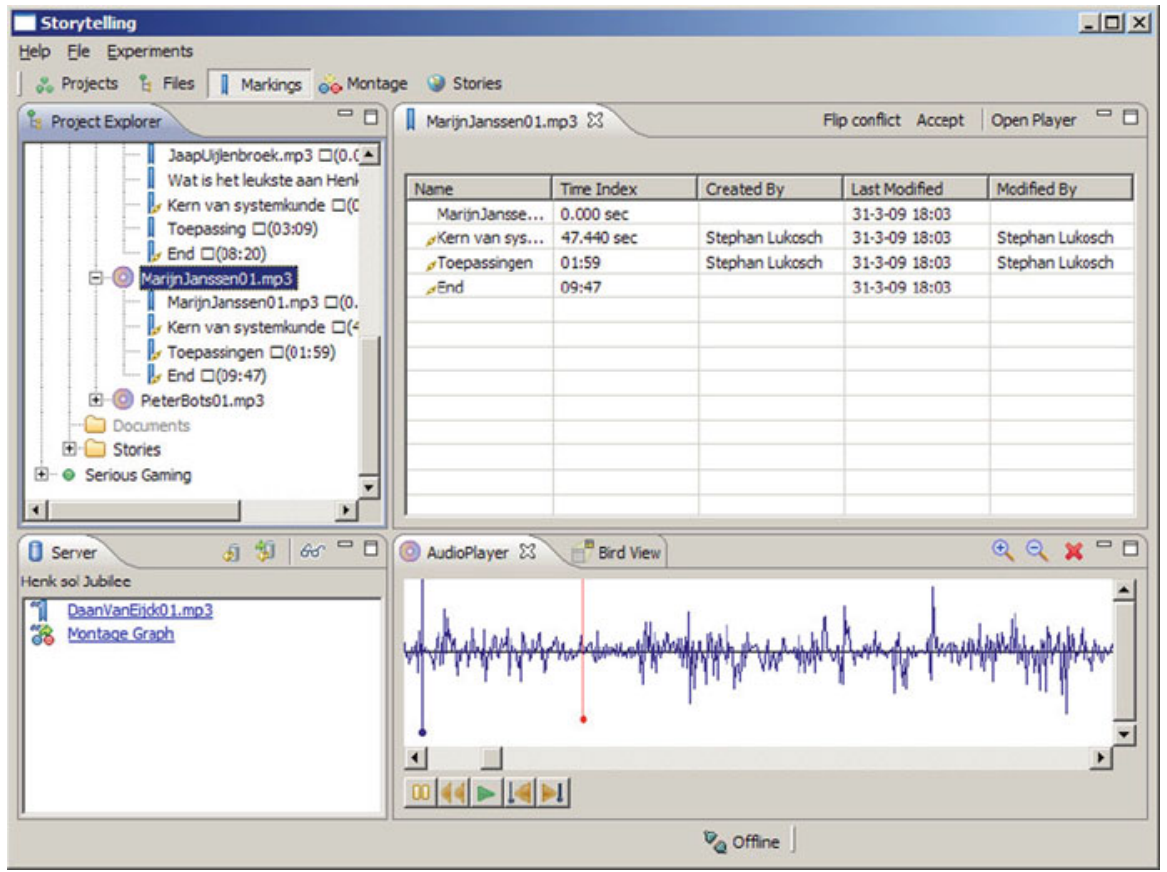

Fig. 2 The marking perspective

\subsection{Linking Audio Recordings}

After setting the explicit as well as the implicit marks, users can now specify different start and end points within an audio recording. However, users still need to be enabled to link different audio recordings. For that purpose, the CASTing client application has to offer appropriate support (R4).

Due to the marks that are associated with each audio recording it is possible to link the original audio recording without modifying it. In non-linear stories, the links between the different audio recordings represent a directed graph. The nodes in the story graph represent the parts of the shared audio recording which are in the story. The directed edges in the graph represent the links between these parts.

Each node is assigned to one audio recording. Users can select one starting mark for the node from the available marks. By allowing only one starting mark, the visualization of the story graph is simplified and more important the users' possibility to follow the story flow is increased. However, as the use of an audio recording is not limited to one node in the story graph, users can use the same audio recording for other nodes with possibly different starting marks in the story graph again. Apart from the starting mark they can use each consecutive mark to add a link to another node. Although there is only one starting mark, there might be several links to other nodes. 


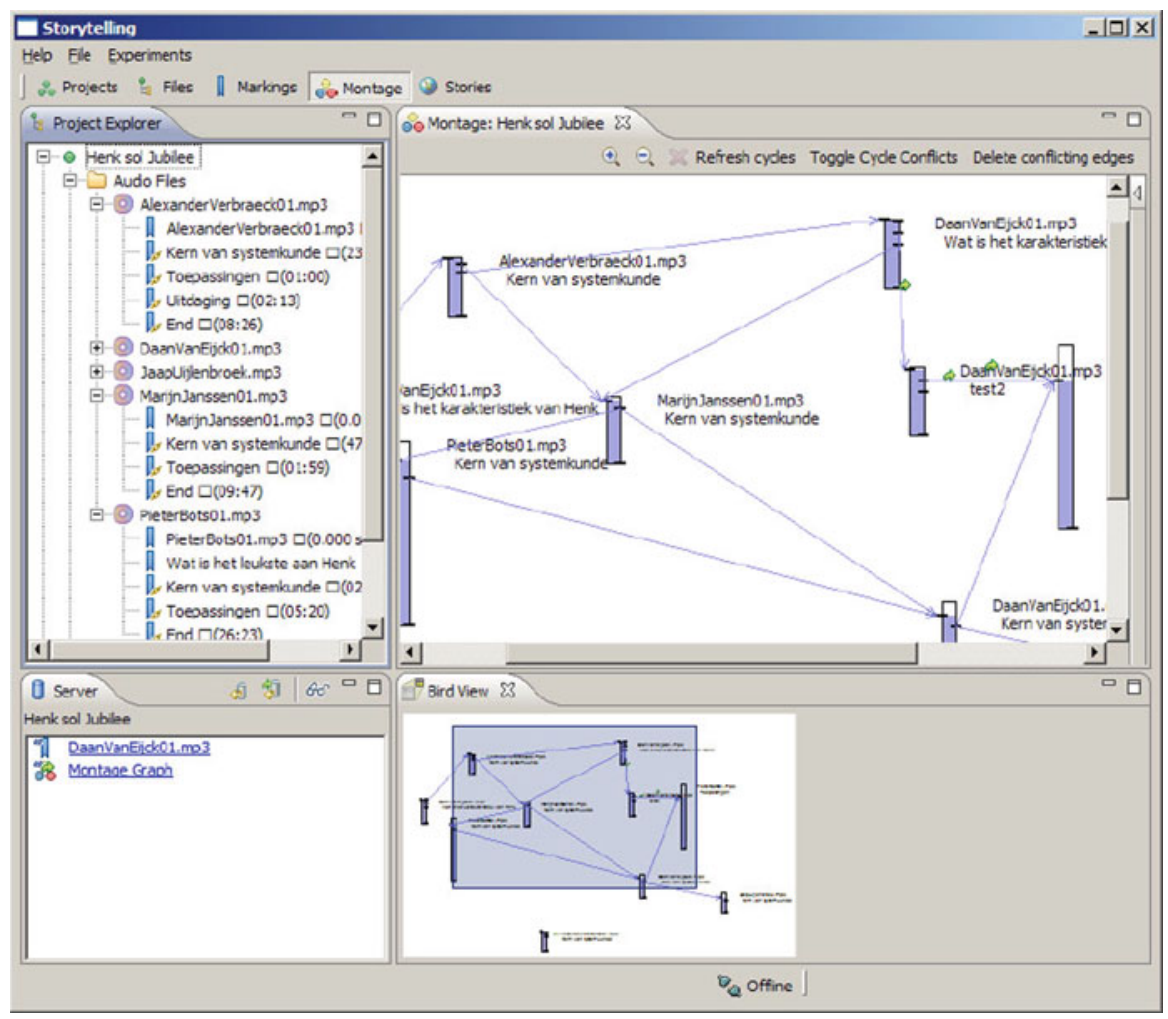

Fig. 3 The story graph perspective

The CASTing client application supports users in collaboratively creating such a story graph. When connected to the server, users can retrieve the most current version of the story graph or synchronize their local changes and thereby update the story graph at the server. By adding nodes and linking nodes, users can create a story graph in which each path between two nodes represents a new story. To ensure that users do not create cycles, the client application only allows links between two marks when this link does not lead to a cycle.

Figure 3 shows the story graph perspective of our CASTing client application. The upper left corner again shows the project explorer with the shared audio recordings and their marks. The lower right corner shows a bird's eye view of the current story graph. The upper right part of the figure shows the current story graph. Each rectangle represents one audio recording. The filled part within the rectangle highlights the part of the audio recording which is used in the story. The mark which is used as starting mark is highlighted by a bar on the left side of the rectangle. Marks which are used to link to other audio recordings are shown as bars on the right side of the rectangle. New nodes can be included by dragging a selected mark on the canvas for the story graph. This mark serves as starting mark for the node. If this is not the implicit starting mark of the audio recording the rectangle is visualized as described above and the name of the starting mark is shown below the name of the audio recording right next to the rectangle. 


\subsection{Publishing a Story}

In this process step, users can select individual paths from the story graph and publish the path as a story in the group's shared workspace in the web portal (R5).

To publish a story, users have to select a starting node and an end node. Based on this specification, the CASTing client application calculates the shortest path between these two nodes. Users can adjust this path by adding new nodes to the story. Whenever such a node is added, the shortest path is recalculated to include this new intermediate node. The current path is always highlighted in a textual description on the left side of the publishing perspective as well as in the story graph by highlighting the included part of each node in a different color. Users can also listen to the current path before it is finally published. When the user finishes the selection of the path, the client application uses the included information to create a continuous audio recording. This audio recording is then uploaded to the shared workspace in the web portal for further processing.

Once a mark or an audio recording with its representing node is included in a story, some restrictions concerning future changes apply. None of these can be deleted or changed. This only becomes possible, when the story is deleted as well. Thereby, we ensure that the basic information for a story is available all the time. Additionally, this fosters users to create alternative stories when they want to comment on a story instead of deleting the disliked parts of a story.

Figure 4 shows a screenshot of the publishing perspective. In this perspective the project explorer is replaced with a textual description of the currently defined story. The textual description starts with a name for the story and a textual description of the story. It contains all nodes in the order as they are included in the story. By selecting a node in the textual description, users can review additional information as the used starting mark or the textual description of the audio recording. It is also possible to listen to the part of the audio recording which is used in the story. The upper right corner contains the story graph in which the currently selected story components are highlighted in a different color. Currently, the local user has selected a story that starts at the 'AlexanderVerbraeck01.mp3' audio recording and then passes the nodes, 'MarijnJanssen01.mp3', 'DaanVanEijk01.mp3', 'PieterBots01.mp3'.

\subsection{Synchronization and Nomadic Work Support}

The CASTing client application stores most items (including audio recordings, marks and nodes and edges of a story graph) both locally and on the server, thereby allowing users to work nomadically and implementing the NOMADIC OBJECTS pattern (Schümmer and Lukosch 2007). However, after performing local changes, users need to synchronize their changes with the latest versions of the group's items and resolve any conflicts that might have occurred (R6).

Figure 5 shows the part of the user interface which allows users to synchronize their local project data with the most recent version on the server. For this purpose, first users have to login to the server and provide their authentication data. The button to the right (1) allows users to download the most recent version from the server. The 


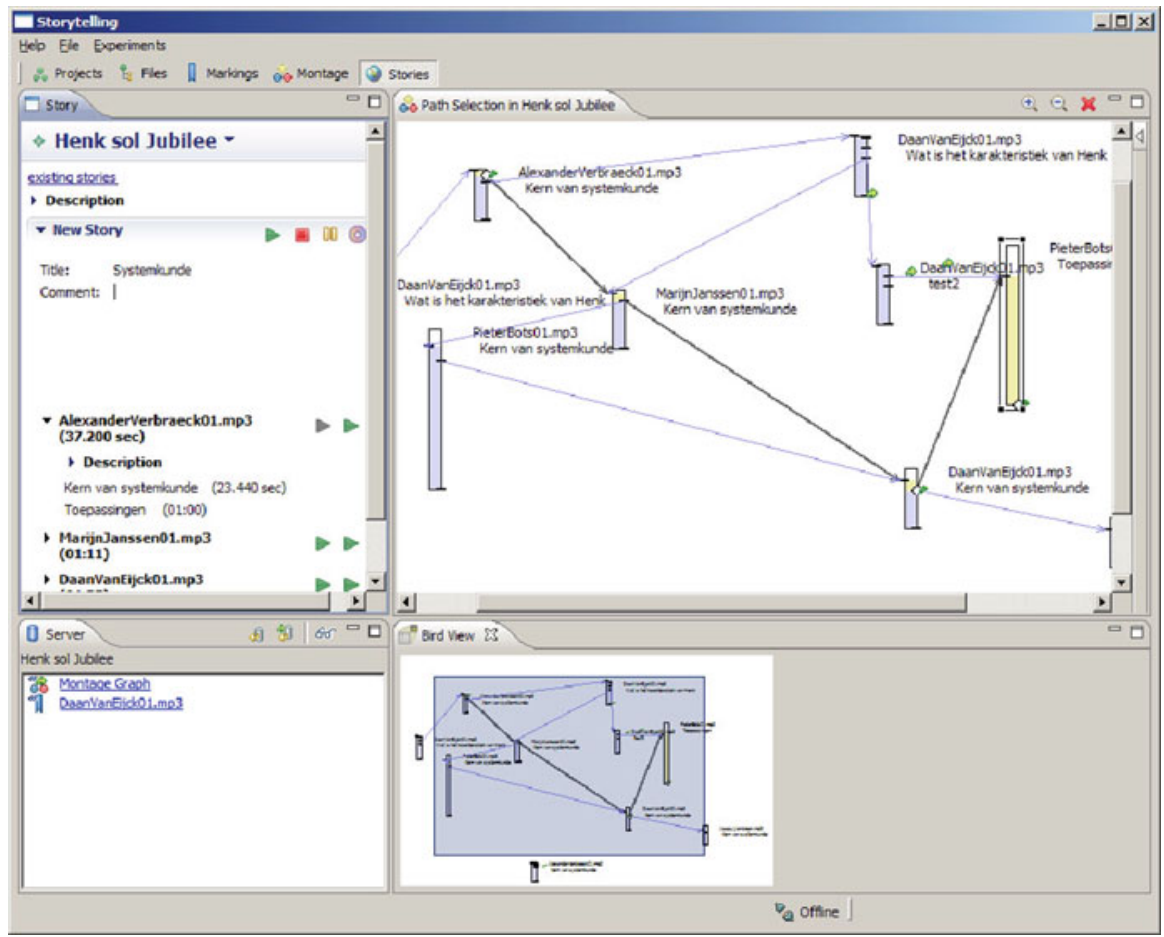

Fig. 4 The publishing perspective

\section{3}

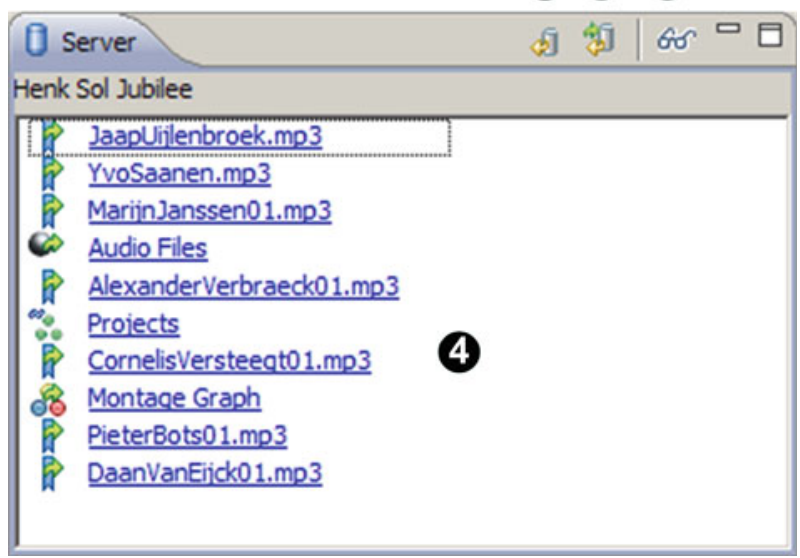

Fig. 5 Server synchronization and change awareness

next button (2) allows users to upload all local changes. Via the glasses button (3) users can highlight the most recent changes in he current perspective, i.e. new nodes, links, marks, files, etc. are equipped with the glasses. Apart from these options, the list 
(4) shows the local as well as the remote changes since the last synchronization. By clicking on the text, the client changes the perspective and allows users to identify the most recent changes in detail. Thereby, the CHANGE INDICATOR pattern (Schümmer and Lukosch 2007) is implemented to achieve group awareness.

After selecting a project and starting the synchronization mechanism, a user is presented with a merged version of local items and shared items. The CASTing client application highlights the group's modifications and displays deleted items in the background, allowing the user to become aware of and comprehend the group's modifications. Furthermore, the client application points out conflicting changes. In these cases, only the latest modifications are shown, but access to the conflicting versions is also offered. The user has to review and resolve these conflicts before being allowed to commit local changes.

As stated previously, once a mark is used in a node, or an audio recording is used in a story, they may not be deleted. Therefore, in some cases the client application restores items deleted by the group. For example, if a user has utilized a mark (deleted by the group) to define a link in the graph, the client application restores the mark and only thereafter the graph may be shared.

\subsection{Community Platform}

As mentioned before, our web portal is based on (Liferay-Enterprise Open Source Portal 2009). It allows users to register, to create a project, to invite project members, to join ongoing projects, to upload and share audio recordings, to communicate via chat or message board, to view who else is currently on line, and to review all project-related information.

Apart from the above functionality, the web portal also supports users in starting story-related discussions, commenting stories, and voting on stories. Voting can be used by a project team to decide which of the alternative threads in the story graph is finally published as podcast to all members of the web portal and thus is available to the public. How such a voting is conducted depends on the chosen strategy within the project team. When the project is created, the voting strategy needs to be chosen. These strategies can vary from using no voting at all, publishing each story that has passed a certain threshold to the more restrictive strategy up to only publish the story with the highest rating from the project team. Such a published podcast can of course be included in a new story project, segmented by marks, linked with other audio recordings, and finally be published again.

As audio as a medium is not easily accessible and the amount of available audio recordings can quickly increase, the CASTing web portal supports collaborative tagging, special awareness mechanisms and searching. Users of the CASTing web portal can collaboratively tag projects, audio clips, documents, stories as well as marks. The tags are user-defined. When a tag cloud is displayed, users can apply different filters to only show the tags for the categories which they currently require. By clicking on tags within a tag cloud the tagged elements are shown in a summary page. Thereby, users can quickly get an overview about the available and most important content within a project. To assess the most recent changes, the CHANGE INDICATOR pattern 


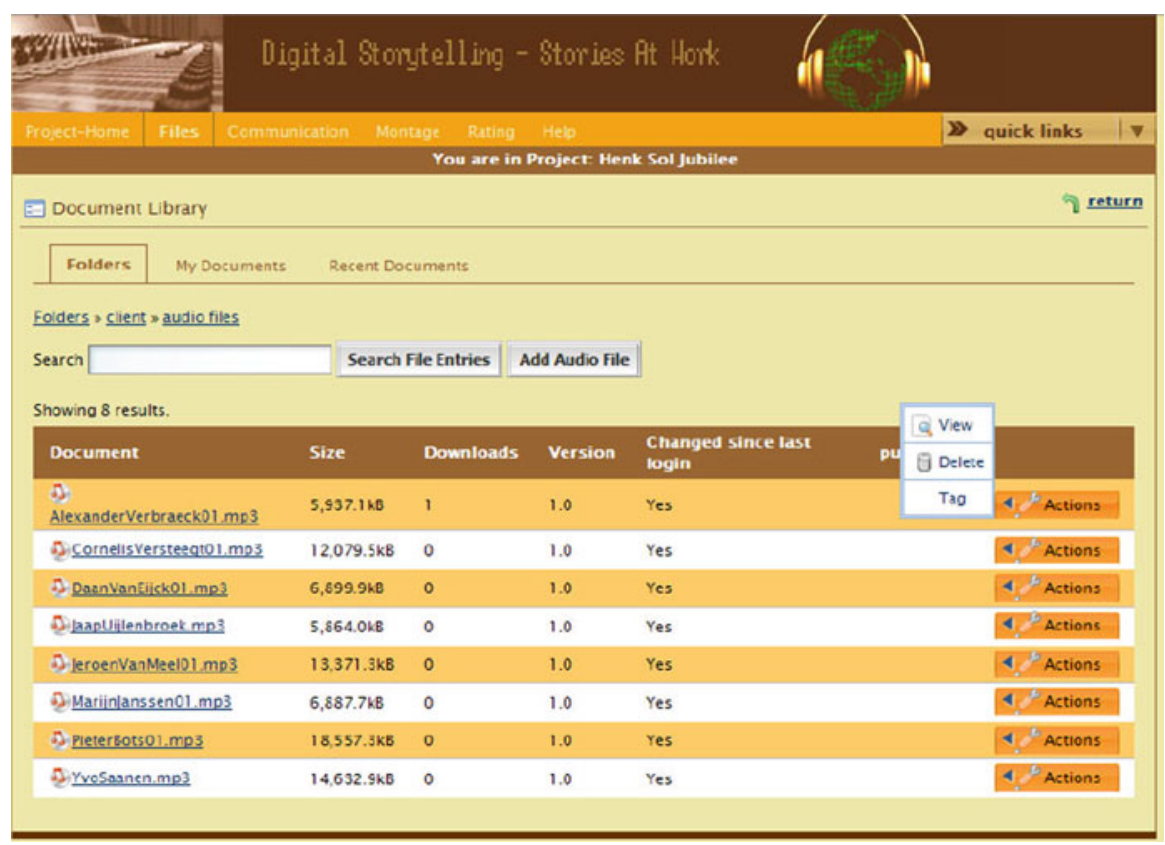

Fig. 6 The web portal

(Schümmer and Lukosch 2007) was implemented. Thereby, the CASTing web portal highlights for each user the most recent changes to the project content. Finally, the search functionality allows users to search within the shared content of a project. The search base includes the filenames, the textual description of audio clips, the name of marks as well tags.

Figure 6 shows a screenshot of the web portal. The screenshot shows the audio library of the 'Henk Sol Jubilee' project. Here, users can access the different audio recordings, download and listen to the audio recordings, add new recordings, tag recordings, or search for recordings. The menu bar on top of the screenshot allows to access further functionality of the web portal, i.e. the project homepage with the corresponding user management functionality, the graphical view of the most recent story graph, as well as the voting functionality which allows users to vote on the published stories.

\section{First Experiences}

In order to validate our design and improve it in further development iterations, the evaluation of our support for collaborative audio-based storytelling is divided into three phases. In the first phase, we conducted functional tests, in order to examine whether our requirements (R1) to (R8) are fulfilled. These tests were all together positive. 
In a second phase, we installed a test environment in our group to conduct usability inspections. This way we had the client application tested by different 3 different groups in the size of 2-3 users. The application cases varied:

- Retelling a well-known fairy tale from memory

- Creation of a podcast episode in an online seminar

- Collection of knowledge about and personal experiences with a specific person

The feedback received from the usability inspections confirmed that our solution allows users to collaboratively produce audio-based stories. Still, we also received a lot of feedback on how to increase the usability of the user interface. A more substantial feedback was that users asked for a better support to assess the content of audio recordings. Users asked for a better tagging support which ranged from simply suggesting tags on the basis of existing tags up to embedding story-related ontologies in the system which then could be used to guide the tagging. We thus continue our work on social tagging functionality. In addition, we will investigate to what extent current technology supports an automatic transcription of audio clips and whether this transcription can be used to automatically index audio clips.

Since the design goal to enable the free and spontaneous development of collectively told stories is central, in particular those areas of application of the informal knowledge management are of interest in the third evaluation phase, which address equal discourse (peer-to-peer) or participation as well as qualification for participation (bottom-up). This comprises collaborative learning scenarios. In addition to this, forms of expert-lay communication play a central role, e.g. in the application of participatory design methods in engineering and informatics, where users are included into product development. Parallels can be drawn to forms of the emancipatory use of media (as aspired by grassroots media), if the goal of participatory design is referred not only to technical artifacts, but also to social and organizational interactions.

In the third phase, the CASTing environment will thus be used in a large company to elicit tacit knowledge on project management and specifically in relation to topics on stakeholder management, risk management and contracting. Here, experts in the field of project management will conduct interviews with project managers from the company. Currently, these interviews are shared within the CASTing environment. After pre-processing the interviews to improve the sound quality, the interviews are marked and tagged. These marked and tagged interviews will serve as seeding data for two workshops on knowledge elicitation. In the first workshop, the experts conducting the interviews will use the enriched interview audio files to elicit best practices. We will then conduct interviews with these experts to compare their traditional techniques with CASTing. In the second workshop, project managers will work on the interviews and try to create structured stories on the selected topics. They are further requested to reflect on the seeding interviews and integrate their own insights into the stories. The resulting stories will then be used to trigger another round of interviews and to enrich lectures on project management.

The project on eliciting tacit knowledge from senior project managers has just started. Nevertheless, this project will allow us to determine if and to what extent the provided support for the process enables collaborative editing and participative production of audio-based stories. A core question we want to answer is whether 
and how it is possible to retrieve the core message from audio-based stories. We also want to evaluate the limitations of audio in dealing with, e.g., ambiguity, anonymity, or inflection. As audio recordings stay and cannot be edited afterwards, the project already revealed new issues in relation to anonymity. Project managers dislike the idea that some stories might be used against them. Thus, the set of people accessing and working with the audio recordings had to be carefully and explicitly selected.

\section{Conclusion}

The risen demand for audio books and the unprompted rise of podcasting shows a comeback of listening. Collaborative audio-based storytelling avails itself of this comeback and utilizes the act of verbally telling stories in groups. In this collaborative act connected to the very roots of storytelling everybody involved becomes a co-teller: by demands, additions, references, interpretation offers, contributions of individual perspectives and much more. The scope of possible areas of application is very broad and spans from knowledge management, collaborative learning, open channels on local radio to user oriented requirement analysis during product development.

All these scenarios are based on common principles, which require a supporting information system. In this paper, we focused on the design and implementation of such a information system. After discussing the body of knowledge in relation to knowledge management and storytelling, we determined the requirements for an information system supporting collaborative audio-based storytelling. When considering the current state of the art, it became clear that there is currently no information system that addresses all of the eight identified requirements. We then presented the CASTing information system. CASTing consists of a client application and a web portal for collaborative audio-based storytelling. CASTing addresses the identified requirements with a five-stage process. In comparison with other tools, CASTing enables users to collaboratively set marks and thus supports collaborative segmenting of shared audio recordings. Furthermore, CASTing supports a collaborative assembly of single audio recordings, which results in a directed graph. Starting from a chosen starting mark, alternative paths, i.e. different versions of a story, can be selected from this graph for publication. Only by this collaborative assembly the alternative representation of events, knowledge, stories or requirements becomes possible as a genuine collaborative act of telling stories. This way collaboratively told stories can mature towards a constant moral stance and informal knowledge management is explicitly supported, as a group of humans agrees stepwise on a common view of things.

Future research will show if other tools are needed in order to reduce the barriers for the production of common stories, to assist collaborative creation, to improve navigation in the time-based audio clips or to retrieve and sustainable disseminate the core message captured in audio-based stories. First experiences have shown that at a first step the possibilities to assess the content of audio recordings have to be improved. Here, we will continue our work on social tagging functionality and explore possibilities to automatically transcribe audio clips and use the transcription to automatically index audio clips. Furthermore, future research will show which informal processes lead to a story and how a group as a whole arranges processes of evaluation and selec- 
tion of knowledge in informal knowledge management. Since this is not determined by our tool, the question arises, how consensus is reached on a relative stable version of a story as well as how long a stable version endures.

\section{References}

Acosta C, Collazos C, Guerrero L, Pino J, Neyem H, Motele O (2004) StoryMapper: a multimedia tool to externalize knowledge. In: Proceedings of the XXIV conference of the Chilean Computer Science Society. IEEE CS Press, pp 133-140

Armstrong DM (1992) Managing by storying around. A new method of leadership. Doubleday, New York Bayon V, Wilson JR, Stanton D, Boltman A (2003) Mixed reality storytelling environments. Virtual Real 7(1):54-63

Beck K (1999) Extreme programming explained. Addison Wesley, Reading

Boyce ME (1995) Collective centring and collective sense-making in the stories and storytelling of one organization. Organ Stud 16(1):107-137

Brown JS, Duguid P (2000) Balancing act: how to capture knowledge without killing it. Harvard Bus Rev 78(3):73-80

Carminatti N, Borges MRS, Gomes JO (2006) Analyzing approaches to collective knowledge recall. Comput Inform 25(6):547-570

Cheng BHC, Atlee JM (2007) Research directions in requirements engineering. In: FOSE '07: future of software engineering 2007. IEEE Computer Society, pp 285-303

Clark BR (1972) The organizational saga in higher education. Adm Sci Q 17(2):178-184

Cognition and Technology Group, Vanderbilt University (1992) An anchored instruction approach to cognitive skills acquisition and intelligence tutoring. In: Regian JW, Schute VJ (eds) Cognitive approaches to automated instruction. Lawrence Erlbaum Associates, Hillsdale pp 135-170

Denning S (2000) The Springboard: how storytelling ignites action in knowledge-era organisation. Butterworth-Heinemann, Boston

Faro A, Giordano D (1998) Storynet: an evolving network of cases to learn information systems design. IEE Proc Softw 145(4):119-128

Gillmor D (2006) We the media: grassroots journalism by the people, for the people. O'Reilly Media, USA

Hampton H, Fayer S (eds) (1990) Voices of freedom: an oral history of the civil rights movement from the 1950s through the 1980s. Bantam Books, New York

Hein G, Jakuska R (2007) Podcast industry. iLabs-Center for Innovation Research, School of Management, University of Michigan-Dearborn

Hevner AR, March ST, Park J, Ram S (2004) Design science in information systems research. MIS Quarterly 28(1):75-105

Howley K (2000) Radiocracy rulz! Microradio as electronic activism. Int J Cult Stud 3(2):256-267

Keil M, Carmel E (1995) Customer-developer links in software development. Commun ACM 38(5):33-44

Lambert J (2008) Digital storytelling: capturing lives, creating community. 2. Life On The Water, Inc., Berkeley

Landry B, Guzdial M (2006a) Learning from human support: Informing the design of personal digital story-authoring tools. In: Proceedings of CODE 2006

Landry BM, Guzdial M (2006b) iTell: supporting retrospective storytelling with digital photos. In: DIS ‘06: proceedings of the 6th ACM conference on designing interactive systems. ACM Press, New York, pp 160-168

Laporti V, Borges MR, Braganholo V (2009) Athena: a collaborative approach to requirements elicitation. Comput Ind 60(6):367-380

Liferay-Enterprise Open Source Portal (2009). http://www.liferay.com

Linde C (2001) Narrative and social tacit knowledge. J Knowl Manage 5(2):160-171

Lukosch S, Klebl M, Buttler T, Hackel M (2009) Eliciting requirements with audio-based collaborative storytelling. In: Kilgour M (ed) Proceedings of the international conference on group decision and negotiation (GDN), pp 99-104

McDrury J, Alterio M (2003) Learning through storytelling in higher education: using reflection and experience to improve learning. Kogan Page Ltd, London 
McWhinney W, Batista J (1988) How remythologizing can revitalize organizations. Organ Dyn 17(2): $46-58$

Melnik G, Maurer F (2004) Direct verbal communication as a catalyst of agile knowledge sharing. In: Agile development conference (ADC'04). IEEE Computer Society, Los Alamitos, pp 21-31

Nyrgen L, Blom B (2001) Analysis of short reflective narratives: a method for the study of knowledge in social workers' actions. Qual Res 1(3):369-384

Ochs E, Capps L (2001) Living narrative. Creating lives in everyday storytelling. Harvard University Press, Cambridge

Ong WJ (1982) Orality and literacy. The technologizing of the word. New accents. Methuen, London [u.a.]

Perret R, Borges MR, Santoro FM (2004) Applying group storytelling in knowledge management. In: Groupware: design, implementation, and use, 10th international workshop, CRIWG 2004, LNCS 3198. Springer, Berlin, pp 34-41

Philips D (2007) Talking books: the encounter of literature and technology in the audio book. Convergence 13(3):293-306

Randall D, Rouncefield M, Hughes JA (1995) Chalk and cheese: Bpr and ethnomethodologically informed ethnography in cscw. In: ECSCW'95: proceedings of the fourth conference on European conference on computer-supported cooperative work. Kluwer, Norwell, pp 325-340

Ready DA (2002) How storytelling builds next-generation leaders. MIT Sloan Manage Rev 43(4):63-70

Röber N, Huber C, Hartmann K, Feustel M, Masuch M (2006) Interactive audiobooks: combining narratives with game elements. In: Technologies for interactive digital storytelling and entertainment, LNCS 4326. Springer, Berlin, pp 358-369

Santoro FM, Borges MR, Pino JA (2009) Acquiring knowledge on business processes from stakeholders' stories. Adv Eng Inform (in press)

Schäfer L, Stauber A, Bokan B (2004) Storynet: an educational game for social skills. In: Technologies for interactive digital storytelling and entertainment, pp 148-157

Schäfer L, Valle C, Prinz W (2004) Group storytelling for team awareness and entertainment. In: NordiCHI '04: proceedings of the third Nordic conference on human-computer interaction. ACM Press, New York, pp 441-444

Schank RC, Fano A, Bell B, Jona M (1994) The design of goal-based scenarios. J Learn Sci 3(4):305-345

Schreyögg G, Geiger D (2005) Reconsidering organizational knowledge: knowledge, skills, and narrations. In: Schreyögg G, Koch J (eds) Knowledge management and narratives. Organizational effectiveness through storytelling. Erich Schmidt Verlag, Berlin pp 291-312

Schümmer T, Lukosch S (2007) Patterns for computer-mediated interaction. Wiley, New York

Shen C, Lesh NB, Vernier F, Forlines C, Frost J (2002) Sharing and building digital group histories. In: CSCW '02: proceedings of the 2002 ACM conference on computer supported cooperative work. ACM, New York, pp 324-333

Snowden D (2000a) The art and science of story or 'are you sitting uncomfortably?' part 1: Gathering and harvesting the raw material. Bus Inf Rev 17(3):147-156

Snowden D (2000b) The art and science of story or 'are you sitting uncomfortably?' part 2: The weft and the warp of purposeful story. Bus Inf Rev 17(4):215-226

Sole D, Wilson DG (2002) Storytelling in organizations. The power and traps of using stories to share knowledge in organizations. Learning Innovations Laboratories, Harvard Graduate School of Education, Cambridge

Spaniol M, Klamma R, Sharda N, Jarke M (2006) Web-based learning with non-linear multimedia stories. In: Advances in web based learning-ICWL 2006, LNCS 4181. Springer, Berlin, pp 249-263

Spring Application Framework (2009). http://springframework.org/

Steiner KE, Moher TG (1992) Graphic storywriter: an interactive environment for emergent storytelling. In: CHI '92: proceedings of the SIGCHI conference on human factors in computing systems. ACM Press, New York, pp 357-364

Stewart TA (1998) The cunning plots of leadership. Fortune 138(5):165-166

Struts Framework (2009). http://struts.apache.org/

Swap W, Leonard D, Shields M, Abrams L (2001) Using mentoring and storytelling to transfer knowledge in the workplace. J Manage Inf Syst 18(1):95-114

Valle C, Prinz W, Borges MRS (2002) Generation of group storytelling in post-decision implementation process. In: Shen W, de Souza JM, Barthés J-PA, Lin Z (eds) The 7th international conference on computer supported cooperative work in design, 2002. IEEE, pp 361-367 
Viégas FB, Boyd D, Nguyen DH, Potter J, Donath J (2004) Digital artifacts for remembering and storytelling: Posthistory and social network fragments. In: Proceedings of the 37 th annual Hawaii international conference on system sciences (HICSS'04), vol 4. IEEE Computer Society, Los Alamitos

VoiceThread (2009). http://voicethread.com/

Zalinger J (2009) Storyfox: a conceptual design for self-reflective storytelling. In: MIT's media in transition 6 (to be presented) 